\title{
REDUCIBILITY OF STANDARD REPRESENTATIONS
}

\author{
BY DAN BARBASCH ${ }^{1}$ AND DAVID A. VOGAN, JR. ${ }^{2}$
}

Let $G$ be a real linear reductive group with abelian Cartan subgroups. Unexplained notation, in general, follows [3 and 6]. Fix a parabolic subgroup $P=M A N$ of $G$ and a representation $\delta$ of $M$ in the limits of the discrete series. The continuous family of representations

$$
\pi(\nu)=\operatorname{Ind}_{P}^{G}(\delta \otimes \nu \otimes 1) \quad\left(\nu \in \hat{A} \cong \mathfrak{a}^{*}\right)
$$

is a typical series of standard representations of $G$. (These are not, in general, unitary since $\nu$ may not be a unitary character of $A$.) In order to apply certain "continuity arguments" in the study of unitary representations of $G$, it is necessary to know for which values of $\nu$ the representations $\pi(\nu)$ is reducible. We sketch here an explicit answer to this question for classical groups. (Our techniques reduce the problem for exceptional groups to a (long) finite calculation.) The continuity arguments mentioned above require a similar understanding of reducibility for some larger class (it is not yet clear what larger class) of induced representations. Some of our techniques also apply to this more general problem.

Write $\bar{\pi}(\nu)$ for the direct sum of the Langlands subquotients of $\pi(\nu)$. These are the irreducible composition factors of $\pi(\nu)$ whose matrix coefficients exhibit the largest possible growth at infinity [1]. (Alternatively [4], they may be characterized by the fact that their restrictions to a maximal compact subgroup contain representations which are as small as possible.) Obviously $\pi(\nu)$ is reducible if and only if at least one of the following conditions holds: $\bar{\pi}(\nu)$ is reducible; or $\pi(\nu)$ has some composition factor not in $\bar{\pi}(\nu)$. We write the second possiblity as $\pi(\nu) \neq \bar{\pi}(\nu)$. Now Knapp and Zuckerman have determined in [2] exactly when the first possibility occurs: $\nu$ must belong to one of finitely many linear subspaces in $\mathfrak{a}^{*}$, which are explicitly described in terms of the inducing representation $\delta$. We must therefore explain when $\pi(\nu) \neq \bar{\pi}(\nu)$.

In writing a Langlands decomposition $P=M A N$, we have implicitly fixed a Cartan involution $\theta$. Choose a $\theta$-stable compact Cartan subgroup $T \subseteq M$ and write $H=T A$ for the corresponding $\theta$-stable Cartan subgroup of $G$. The representation $\delta$ determines (up to conjugacy under $W(M, T)$ ) a positive root system $\Delta^{+}(\mathfrak{m}, \mathfrak{t})$ and a Harish-Chandra parameter $\lambda \in \mathfrak{t}^{*}$. Put

$$
\begin{gathered}
\bar{\gamma}=(\lambda, \nu) \in \mathfrak{t}^{*}+\mathfrak{a}^{*} \cong \mathfrak{h}^{*}, \\
R(\delta \otimes \nu)=\{\alpha \in \Delta(\mathfrak{g}, \mathfrak{h}) \mid\langle\check{\alpha}, \bar{\gamma}\rangle \in \mathbf{Z}\} ;
\end{gathered}
$$

as usual, $\not{\alpha}$ denotes the coroot $2 \alpha /\langle\alpha, \alpha\rangle$.

Received by the editors May 18, 1984 and, in revised form, July 12, 1984.

1980 Mathematics Subject Classification. Primary 22E46.

${ }^{1}$ Supported by an NSF grant and a Rutgers Research Council grant.

${ }^{2}$ Supported in part by NSF grant MCS-8202127. 
The root system $R(\delta \otimes \nu)$ has several additional structures:

(1) $\theta$ acts on $R(\delta \otimes \nu)$.

(2) Each $\theta$-fixed (that is, imaginary) root is either compact or noncompact.

(3) Each $(-\theta)$-fixed (that is, real) root either does or does not satisfy a "parity condition" $[\mathbf{3}]$.

(4) There is a decomposition

$$
R(\delta \otimes \nu)=R^{++}(\delta \otimes \nu) \cup R_{0}(\delta \otimes \nu) \cup R^{--}(\delta \otimes \nu)
$$

of the roots according to whether their inner products with $\bar{\gamma}$ are positive, zero, or negative.

(5) There is a distinguished choice $\Delta^{+}(\mathfrak{m}, \mathfrak{t})$ of positive imaginary roots.

Fix a nonzero weight $\phi \in \mathfrak{a}^{*}$ of $\mathfrak{a}$ in $\mathfrak{g}$, and set

$$
R(\delta \times \nu)^{\phi}=\left\{\alpha \in R(\delta \otimes \nu)|\alpha|_{\mathfrak{a}} \in \mathbf{R} \cdot \phi\right\} .
$$

This root system inherits all the extra structure of $R(\delta \otimes \nu)$. Choose a positive root system $R_{0}^{+}(\delta \otimes \nu)^{\phi}$ so that

(a) $R_{0}^{+}(\delta \otimes \nu)^{\phi} \supseteq \Delta^{+}(\mathfrak{m}, \mathfrak{t}) \cap R_{0}(\delta \otimes \nu)$.

(b) If $\alpha \in R_{0}(\delta \otimes \nu)^{\phi}$ and $(-\theta \alpha) \in R^{++}(\delta \otimes \nu)^{\phi}$, then $\alpha \in R_{0}^{+}(\delta \otimes \nu)$.

(c) If $\alpha$ and $-\theta \alpha$ are distinct elements of $R_{0}(\delta \otimes \nu)^{\phi}$, then both belong to $R_{0}^{+}(\delta \otimes \nu)$, or neither does.

Define

$$
\begin{gathered}
{ }^{\mathbf{R}} R^{+}(\delta \otimes \nu)^{\phi}=R^{++}(\delta \otimes \nu)^{\phi} \cup R_{0}^{+}(\delta \otimes \nu)^{\phi}, \\
\Pi={ }^{\mathbf{R}} \Pi(\delta \otimes \nu)=\text { simple roots of }{ }^{\mathbf{R}} R^{+}(\delta \otimes \nu)^{\phi} .
\end{gathered}
$$

If $-\theta$ preserves ${ }^{\mathbf{R}} R^{+}(\delta \otimes \nu)^{\phi}$, define $C(\delta \otimes \nu)^{\phi}$ to be the empty root system. Otherwise, we can write

$$
\phi=\sum_{\alpha \in \Pi} n_{\alpha} \alpha,
$$

with $n_{\alpha}$ a nonnegative rational number. We define

$$
\begin{gathered}
\Pi_{\mathrm{crit}}={ }^{\mathbf{R}} \Pi(\delta \otimes \nu)_{\mathrm{crit}}^{\phi}=\left\{\alpha \in \Pi \mid n_{\alpha} \neq 0\right\}, \\
C(\delta \otimes \nu)^{\phi}=\text { span of } \Pi_{\mathrm{crit}},
\end{gathered}
$$

the critical root system.

Proposition [5]. There is a connected simple group $\tilde{G}$ with parabolic subgroup $\tilde{P}=\tilde{M} \tilde{A} \tilde{N}, \tilde{\delta} \in \hat{\tilde{M}}, \tilde{\nu} \in \tilde{\mathfrak{a}}^{*}$, etc., all unique up to isomorphism, such that

(a) $\operatorname{dim} \tilde{A}=1$.

(b) $\tilde{\pi}(\tilde{\nu})=\operatorname{Ind} \tilde{G}_{\tilde{P}}^{\tilde{S}}(\tilde{\delta} \otimes \tilde{\nu} \otimes 1)$ has integral infinitesimal character.

(c) $\Delta(\tilde{\mathfrak{g}}, \tilde{\mathfrak{h}})=R(\tilde{\delta} \otimes \tilde{\nu}) \cong C(\delta \otimes \nu)^{\phi}$, the isomorphism preserving the additional structures (1)-(5) described above.

The critical root system $C(\delta \otimes \nu)^{\phi}$ is said to be of reducible type if the representation $\tilde{\pi}(\tilde{\nu})$ described by the proposition is reducible. 
THEOREM. Let $\pi(\nu)=\operatorname{Ind}_{P}^{G}(\delta \otimes \nu)$ be a standard representation as described above. Then $\pi(\nu) \neq \bar{\pi}(\nu)(\pi(\nu)$ is distinct from its Langlands subquotients) if and only if there is a nonzero weight $\phi$ of $\mathfrak{a}$ in $\mathfrak{g}$ such that the attached critical root system $C(\delta \otimes \nu)^{\phi}$ (defined above) is nonempty and of reducible type.

This was largely proved (implicitly) in [3]. Our solution to the reducibility problem (for classical groups) consists of a list of all critical root systems (together with the additional structures (1)-(5)) which are not of reducible type. For complex groups and $\mathrm{GL}(n, \mathbf{R})$, there are no such irreducible critical root systems (except the empty one), so the theorem reduces to results of Zhelobenko [9] and Speh [8], respectively. Kostant's results [7] on reducibility of spherical series may be interpreted as describing certain irreducible critical root systems corresponding to the large region of irreducibility round $\nu=0$. The group $\tilde{G}$ of the Proposition in these cases has real rank one. This already accounts for many of the irreducible critical root systems. Most of the rest correspond to $\tilde{G}$ of real rank 2 .

We also give tables describing the smallest real $\nu$ for which $\pi(\nu)$ is reducible when $\operatorname{dim} A=1$ and use these to study unitarizability of $\bar{\pi}(\nu)$ in that case. Details and proofs will appear elsewhere.

\section{REFERENCES}

1. A. Borel and N. Wallach, Continuous cohomology, discrete subgroups, and representations of reductive groups, Princeton Univ. Press, Princeton, N.J., 1980.

2. A. W. Knapp and G. Zuckerman, Classification of irreducible tempered representations of semisimple groups, Ann. of Math. (2) 116 (1982), 389-455.

3. B. Speh and D. Vogan, Reducibility of generalized principal series representations, Acta Math. 145 (1980), 227-299.

4. D. Vogan, The algebraic structure of the representations of semisimple Lie groups. I, Ann. of Math. (2) 109 (1979), 1-60.

5. - Irreducible characters of semisimple Lie groups. IV: Character-multiplicity duality, Duke Math. J. 49 (1982), 943-1073.

6. __ Representations of real reductive Lie groups, Birkhauser, Boston, 1981.

7. B. Kostant, On the existence and irreducibility of certain series of representations, Bull. Amer. Math. Soc. 75 (1969), 627-642.

8. B. Speh, Some results on principal series for GL $(n, \mathbf{R})$, Ph.D. Diss., MIT, June 1977.

9. D. P. Zhelobenko, The analysis of irreducibility in the class of elementary representations of a complex semisimple Lie group, Math. USSR Izv. 2 (1968), 105-128.

Department of Mathematics, RUtgers University, NeW Brunswick, NEW JERSEY 08903

Department of Mathematics, Massachusetts institute of TechnolOGY, CAMBRIDGE, MASSACHUSETTS 02139 